\title{
THE PHYSICAL MEANING OF PHASE COEFFICIENTS
}

\author{
J. VEVERKA \\ Cornell University
}

\begin{abstract}
The question of what information about an asteroid's surface is contained in a measurement of the phase coefficient between phase angles of $10^{\circ}$ and $30^{\circ}$ is examined in detail. Contrary to some past claims it is shown that absolute reflectivities cannot be derived from phase coefficients. Furthermore, typical asteroid phase coefficients cannot be interpreted unambiguously. This is because the observed phase coefficient may depend as much on the photometric properties of an individual surface element as on the degree of large-scale surface roughness, and these two effects are impossible to separate if only disk integrated measurements are available. The wavelength dependence of asteroid phase coefficients should be small and should contain little information about the surface. In the case of irregular asteroids with macroscopically rough surfaces, the importance of large-scale shadowing, and hence the observed phase coefficient, will depend on the aspect of the asteroid. In such cases, therefore, phase coefficients must be carefully defined to be meaningful. It should be possible, in some cases, to estimate the relative surface roughness of two quasi-spherical asteroids by combining photometric and polarimetric observations. For example, if the two asteroids have almost identical polarization curves but quite different phase coefficients, it is likely that the asteroid with the larger phase coefficient has a macroscopically rougher surface.
\end{abstract}

One of the aims of asteroid photometry is to obtain information about the physical characteristics, such as texture, composition, and large-scale roughness, of asteroid surfaces. In this paper I wish to concentrate on a single aspect of asteroid photometry and consider in detail what information can be derived from observed phase coefficients. For instance, is it possible, as Bell (1917), Stumpff (1948), Widorn (1964), and recently Gehrels et al. (1970) have tried to do, to determine the absolute reflectivities of asteroids in this way?

I will use the term "phase coefficient" in a restricted sense. From Earth, few asteroids can be observed at phase angles larger than $30^{\circ}$. Also, at very small phase angles an additional surge in brightness (the "opposition effect") is usually present (Gehrels, 1956, 1967). The details of this opposition surge contain important information about the surface texture (Hapke, 1963; Irvine, 1966), but few asteroids have been observed at sufficiently small phase angles to determine accurately this part of their phase curves. I will therefore use the term "phase coefficient" to mean the slope (in magnitudes per degree of phase) of the observed phase curve between $10^{\circ}$ and $30^{\circ}$. The problem of 
understanding the physical implications of this quantity (which I will denote by $\beta$ ) can be divided into two parts:

(1) To adequately describe the scattering properties of an individual small element of the surface of a typical asteroid

(2) To determine what additional effects are introduced by shadowing due to large-scale roughness

These two questions are dealt with in turn in the next two sections.

\section{THE SCATTERING PROPERTIES OF A SMALL SURFACE ELEMENT}

Observational evidence suggests that the surface of a typical asteroid is similar to that of the Moon; that is, microscopically rough and intricate, and made up largely of a dark material in which multiple scattering is not dominant. The scattering properties of such surfaces have been considered by Irvine (1966); his model gives an exact treatment of the scattering properties of a dark, particulate layer under the following assumptions:

(1) All particles are spherical and of uniform radius.

(2) The particles are large enough that shadowing can be dealt with in terms of geometric optics.

(3) The particles are dark enough for multiple scattering to be negligible.

When a parallel beam of light is incident on an element of such a surface, at an angle $i$, the specific intensity $I$ of the light scattered at an angle $\epsilon$ (making a phase angle $\alpha$ with the incident direction) is given by

where

$$
I(i, \epsilon, \alpha) \propto\left[\bar{\omega}_{0} \Phi(\alpha)\right] \frac{\cos i}{\cos i+\cos \epsilon} S(i, \epsilon, \alpha ; D)
$$

$$
\begin{aligned}
\bar{\omega}_{0} & =\text { scattering albedo of a single particle } \\
\Phi(\alpha) & =\text { phase function of a single particle }
\end{aligned}
$$

$\mathrm{S}(i, \epsilon, \alpha ; D)=$ Irvine shadowing function for the surface

The parameter $D$ is related to the compaction of the surface as follows. If $\rho$ is the mean density of a macroscopic volume element of the surface, and $\rho_{0}$ is the mean density of a single particle, then

$$
D \equiv \frac{3}{4 \pi} \frac{\rho}{\rho_{0}}
$$

For uniform, equally hard spheres, $D$ cannot exceed 0.176 (Beresford, 1969). For the Moon's top surface, Hapke (1963) estimates $\rho / \rho_{0} \cong 0.1$, which corresponds to $D=0.024$.

Using the equations given by Irvine, it is easy to show that $S(i, \epsilon, \alpha ; D)$ does not depend strongly on either $i$ or $\epsilon$ individually, so that

$$
S(i, \epsilon, \alpha ; D) \simeq S(\alpha, D)
$$


Therefore equation (1) may be rewritten as

$$
I(i, \epsilon, \alpha) \sim \widetilde{\omega}_{0} \frac{\cos i}{\cos i+\cos \epsilon} f(\alpha, D)
$$

where $f(\alpha, D) \equiv S(\alpha, D) \Phi(\alpha)$.

Although this equation is based on simplifying assumptions, it does adequately represent laboratory measurements on dark, microscopically rough surfaces. Furthermore, it holds even for surfaces in which the individual particles are not physically separate but are fused together as, for example, in furnace slag.

The validity of equation (2) can be easily tested for any surface in the laboratory by making measurements of $I(i, \epsilon, \alpha)$ as a function of $\alpha$ at a series of fixed values of $\epsilon$; say at $\epsilon=0^{\circ}, 30^{\circ}$, and $60^{\circ}$. From each set of measurements corresponding to a given $\epsilon$, an empirical $f(\alpha, D)$ can be determined using equation (2). If this equation is applicable to the surface, all the $f(\alpha, D)$ values so obtained will be identical.

Such a test is carried out, using measurements on a sample of dark furnace slag (Halajian, 1965), in figure 1 where all the $f(\alpha, D)$ values have been normalized to unity at $\alpha=10^{\circ}$. Because a single $f(\alpha, D)$ is indicated, equation (2) appears to be valid for this surface, even though this surface is not "particulate" in the usual sense. This test can be carried out with equal success for dark surfaces which are particulate in the normal sense. In fact, Halajian

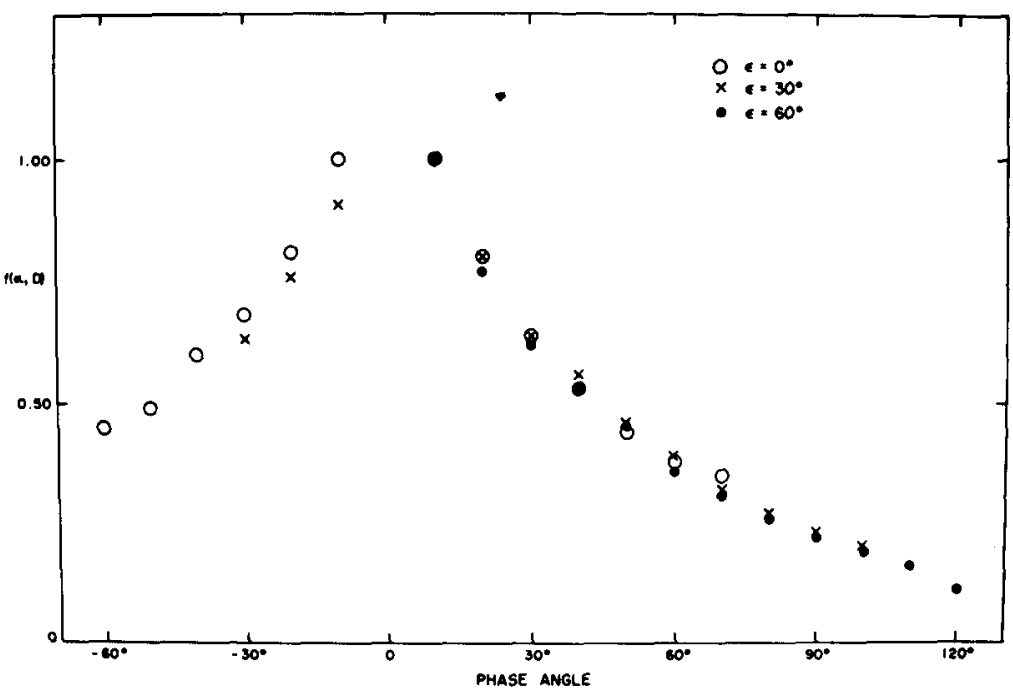

Figure 1.-The $f$ function for a layer of dark furnace slag, from measurements in $V$ by Halajian (1965). This sample has a normal reflectivity of 0.09 and photometric properties very similar to those of the lunar surface. The function is normalized to unity at $\alpha=10^{\circ}$. 
(1965) found that many particulate surfaces (volcanic cinders, for example) have values of $f(\alpha, D)$ almost identical to that shown in figure 1, which incidentally, is very similar to that of the lunar surface.

I will now show that the $f(\alpha, D)$ shown in figure 1 can be adequately reproduced using the Irvine model. In doing this, it is convenient to choose for $\Phi(\alpha)$, the one parameter family of single particle phase functions introduced by Henyey and Greenstein (1941),

$$
\Phi_{\mathrm{HG}}(\alpha, G)=\frac{1-G^{2}}{\left(1+G^{2}-2 G \cos \alpha\right)^{3 / 2}}
$$

The parameter $G \equiv\langle\cos \alpha\rangle$ describes the nature of the scattering. For $G=+1$, there is complete backscattering; for $G=-1$, complete forwardscattering; and for $G=0$ the scattering is isotropic.

The measured $f(\alpha, D)$ shown in figure 1 can only be matched for a very small range of $G(0.30$ to 0.35$)$ (fig. 2). This indicates that effectively the individual particles are slightly backscattering, a result to be expected for large, opaque particles with rough surfaces. In figure 2 , a reasonable choice of $D=0.03$ is used, but the conclusions do not depend strongly on the value of $D$.

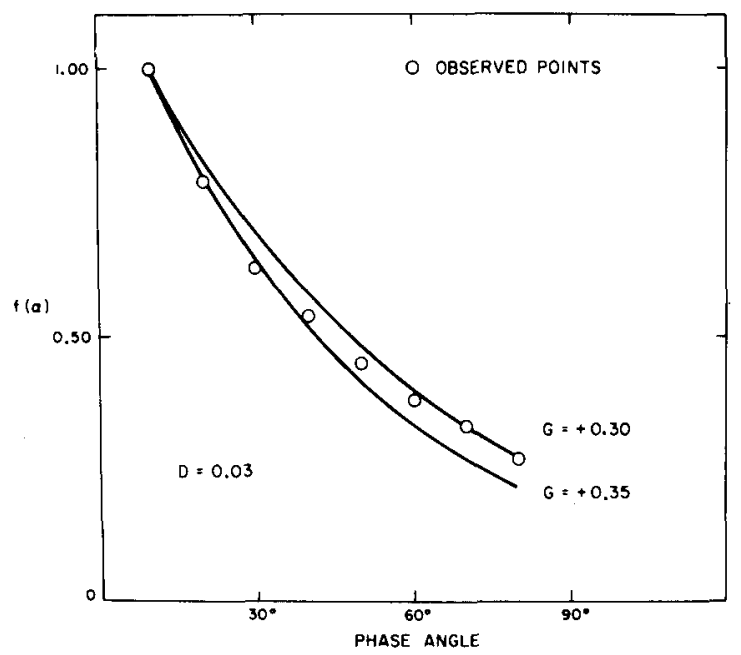

Figure 2.-Comparison of the $f$ function of figure 1 with two theoretical predictions using the Irvine model and a Henyey-Greenstein phase function. The points represent the mean values of $f$ at each phase angle, taken from figure 1 .

I conclude that the Irvine model is adequate for describing the scattering properties of dark, microscopically intricate surfaces. Furthermore, it seems immaterial whether the particles of the surface are physically free or fused together. 


\section{THE EFFECTS OF LARGE-SCALE ROUGHNESS: MACROSCOPIC SHADOWING}

Unfortunately, the general problem of shadowing on a randomly rough two-dimensional surface is extremely complicated. Ideally, one wishes to know for each angle of illumination and each angle of observation what parts of the surface are both illuminated and seen. The surface can be specified statistically in terms of the height deviations from an arbitrary mean level or in terms of the distribution of surface slopes. So far, solutions exist only for onedimensional surfaces (for example Beckmann, 1965, and Saunders, 1967), and I will therefore use a contrived, but convenient model, first introduced by Hämeen-Anttila et al. (1965). In this model the surface is assumed to be bounded on top by a plane that is punctured by countless paraboloidal craters, whose axes of revolution are perpendicular to the plane. The shape of a crater is determined by the parameter $Q \equiv H / R$, where $H$ is the crater depth and $R$ is its radius at the top level.

To study the effects of large-scale shadowing on the photometric properties of asteroids, it is convenient to first consider a model planet that is spherical and completely covered with paraboloidal craters of shape $Q$. (It is assumed that the craters do not overlap.) As $Q$ increases from zero, so does the roughness of the model planet. The rms slope of such a surface is given by

$$
\theta_{\mathrm{rms}}=\arctan \frac{2 Q}{3}
$$

and $Q$ is related to the maximum surface slope by the relation

$$
Q=\frac{\tan \theta_{\max }}{2}
$$

For $\theta_{\max } \leqslant 35^{\circ}$, for example, $Q \leqslant 0.35$.

It is implicitly assumed in the model that, on the one hand, the number of craters per resolution element is very large, whereas on the other hand, each crater is large enough to contain a large number of individual scattering elements. Also, the surface reflectivity is assumed to be low enough that shadows are not affected by multiple scattering.

To determine the total amount of light $j(\alpha)$ scattered by the model planet toward Earth at a phase angle $\alpha$, an integration over the illuminated part of the disk must be performed:

$$
j(\alpha) \propto \iint \bar{I} \cos \epsilon d \sigma
$$

where $\cos \epsilon d \sigma$ is the projected area of the surface element $d \sigma$, and $\bar{I}$ is the effective specific intensity of the light scattered by that element toward Earth. Numerically, this process is conveniently carried out by the method of Horak (1950) in which the integration is replaced by a weighted sum over a grid of points covering the illuminated part of the disk. At each point of this grid, $\bar{I}$ is 
found by calculating the mean specific intensity of the light scattered from a paraboloidal crater (see Hämeen-Anttila et al., 1965, for details), each element of which scatters according to Irvine's law.

Clearly, the $j(\alpha)$ calculated in this way for a surface with $Q>0$ will be less than that found when $Q=0$ at all phase angles $\alpha>0$. We will, in fact, have the following relationship:

$$
j(\alpha, Q)=j(\alpha, 0) \Sigma(\alpha, Q)
$$

where $\Sigma(\alpha, Q)$ is a macroscopic shadowing function that depends only on $\alpha$ and $Q$ (and, of course, on the model of surface roughness) but not on $f(\alpha, D)$. Thus, the effective scattering law for the rough model planet may be considered to be

$$
I_{R}(i, \epsilon, \alpha)=I(i, \epsilon, \alpha) \Sigma(\alpha, Q)
$$

where $I(i, \epsilon, \alpha)$ is given by equation (1) and $\Sigma(\alpha, Q)$ can be determined by the calculation described above. For a macroscopically smooth planet $(Q=0)$, $\Sigma(\alpha, 0)=1$ for all $\alpha$ and

$$
I_{R}(i, \epsilon, \alpha)=I(i, \epsilon, \alpha)
$$

The values of $\Sigma(\alpha, Q)$ for this model, found using either a 36 or 100 point grid over the illuminated part of the planet and a 2500 point grid over each crater, are shown in figure 3 . The numerical accuracy of these values is better than 1 percent. The results of figure 3 can now be used to study the effects of large-scale surface roughness on the photometric parameters of the model planet once $f(\alpha, D)$ is specified. Because the $f(\alpha, D)$ shown in figure 1 is very

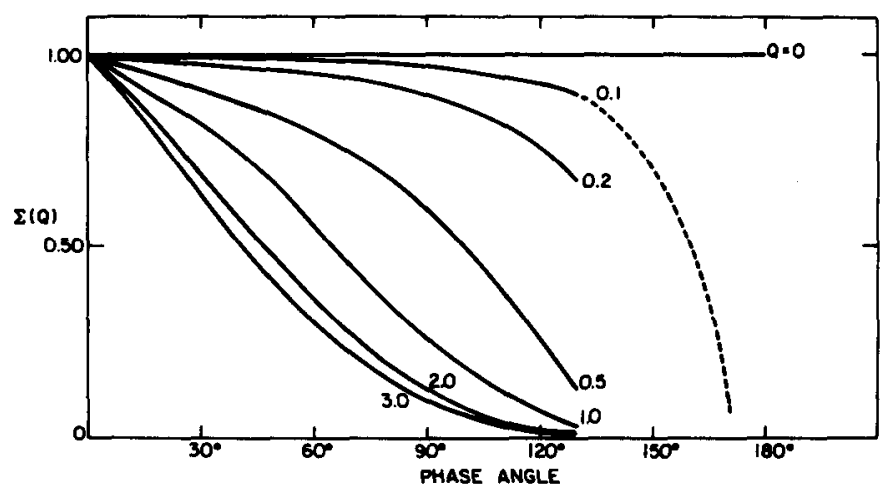

Figure 3.-The macroscopic shadowing function $\Sigma(Q)$ versus phase angle $\alpha$ for various values of $Q$. The nature of $\Sigma$ depends on the specific model of large-scale surface roughness used (in this case the surface is assumed to be covered with paraboloidal craters), but is independent of $f(\alpha, D)$. Note that beyond $Q=2$, increasing the surface roughness produces little change in $\Sigma$. Values of $\Sigma(Q)$ were calculated for $\alpha=0^{\circ}, 10^{\circ}$, $20^{\circ}, 50^{\circ}, 90^{\circ}, 130^{\circ}$, and $170^{\circ}$; for all $Q>0, \Sigma\left(170^{\circ}, Q\right)$ was found to be less than 0.001 . 
similar to that of the Moon, it is of interest to use it in these calculations. For this purpose, it may be extended linearly (on a magnitude scale) from $\alpha=10^{\circ}$ to $\alpha=0^{\circ}$; that is, at $0.026 \mathrm{mag} / \mathrm{deg}$, thus in effect neglecting any opposition effect. Values of the phase coefficient $\beta$ (between $\alpha=10^{\circ}$ and $\alpha=30^{\circ}$ ) and of the phase integral

$$
q \equiv 2 \int_{0}^{\pi} \frac{j(\alpha, Q)}{j(0, Q)} \sin \alpha d \alpha
$$

for this model planet are shown in figure 4 as functions of the roughness parameter $Q$. The phase coefficient is seen to increase significantly as the surface gets rougher until about $Q=2$; for larger values of $Q$ the additional increase in $\beta$ is slight. The phase integral, on the other hand, decreases appreciably as $Q$ increases, but again a leveling off occurs beyond $Q \cong 2$. Note that the phase coefficient $\beta$ of the disk integrated light is related to the laboratory phase coefficient $\beta_{\mathrm{lab}}$, the slope of $f(\alpha, D)$ (on a magnitude scale), by the relation

$$
\beta=\beta_{\mathrm{lab}}+\beta_{\mathrm{ls}}
$$

where $\beta_{\mathrm{Is}}$ is the phase coefficient of a Lommel-Seeliger planet (that is, a planet with $Q=0$ and $f(\alpha, D)=1$ ). Between $\alpha=10^{\circ}$ and $\alpha=30^{\circ}, \beta_{1 \mathrm{~s}} \cong 0.006$

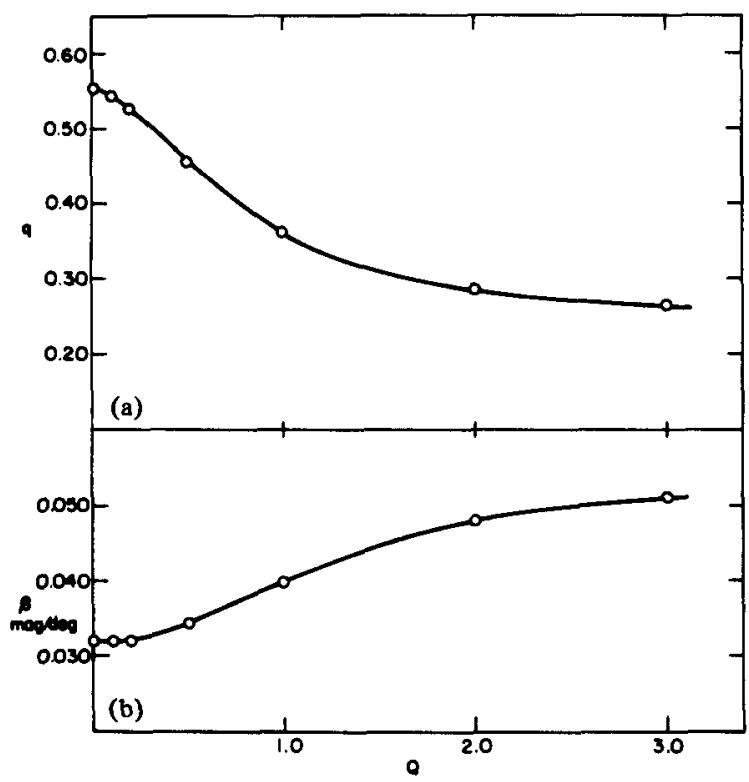

Figure 4.-(a) The phase integral $q$ of the model planet versus the surface roughness (represented by the parameter $Q$ ). The $f$ function shown in figure 1 extrapolated to $\alpha=0^{\circ}$ as described in the text was used in this calculation. (b) The corresponding variation of the phase coefficient $\beta$ measured between $\alpha=10^{\circ}$ and $\alpha=30^{\circ}$. 
$\mathrm{mag} / \mathrm{deg}$. Hence, because over the same interval of phase angles, $\beta_{\mathrm{lab}}=0.026$ $\mathrm{mag} / \mathrm{deg}$ for the surface of figure $1, \beta=0.032 \mathrm{mag} / \mathrm{deg}$ for $Q=0$ in figure 3 .

For a scattering law of type (1), the geometric albedo $p$ of the model planet is independent of $Q$.

Thus, for the above model, it can be concluded that-

(1) Large-scale surface roughness has a strong effect on both the phase integral and the phase coefficient, but none on the geometric albedo.

(2) From equation (1) it follows that the phase coefficient is independent of the single particle albedo $\bar{\omega}_{0}$, but the geometric albedo is not.

(3) Therefore, in view of conclusions (1) and (2), there can be, in general, no correlation between $\beta$ and $p$.

(4) Within the framework of this model, $\beta$ and $q$ are independent of wavelength, unless $\Phi(\alpha)$ has a wavelength dependence. But because it is assumed that the particles of the model surface are opaque and large compared to the wavelength, the wavelength dependence of $\Phi(\alpha)$ will be small.

\section{SOME RELEVANT LABORATORY RESULTS}

Laboratory work with dark, microscopically complex surfaces (Halajian, 1965; Halajian and Spagnolo, 1966) is in accord with these conclusions. Even in the laboratory, when macroscopic shadowing is not important, no general correlation between $\beta_{\text {lab }}$ and the surface reflectivity is found. Also, the observed wavelength dependence of $\beta_{1 a b}$ is very small, but there is an interesting trend for $\beta_{\mathrm{lab}}$ to decrease slightly with increasing wavelength. Because the reflectivity of the samples used in this work tends to increase slightly with increasing wavelength, this suggests that the breakdown of the Irvine model is at least in part due to the increased importance of multiple scattering at longer wavelengths. Multiple scattering makes it easier for light to escape from the surface; this effect is relatively more important at large phase angles because it is then more difficult for singly scattered photons to escape from within the surface. Thus multiple scattering helps to get relatively more light out of the surface at large phase angles than near opposition. This tends to make phase coefficients smallest at those wavelengths at which multiple scattering is most important; that is, usually in the red portion of the spectrum. But for dark surfaces this effect is very small.

Laboratory work such as that referred to above (Halajian, 1965; Halajian and Spagnolo, 1966) shows conclusively that no mineralogical information is contained in phase coefficients; at best one can distinguish materials in which multiple scattering is dominant from those in which it is negligible. In addition, this work shows that away from opposition $\left(\alpha>10^{\circ}\right)$ phase coefficients contain no information about whether a surface is particulate. For example, as already noted, both particulate samples of volcanic cinders and solid samples of 
furnace slag reproduce the lunar photometric function in $V$ equally well as phase angles larger than a few degrees.

\section{A SERIOUS COMPLICATION: NONSPHERICAL ASTEROIDS}

A serious complication in interpreting phase coefficients is that many asteroids are not even approximately spherical. What can be said about the brightness variations with phase of an irregular asteroid whose aspect changes with time? Clearly, as the aspect changes, so will the importance of large-scale shadowing.

Consider the following idealized example of an ellipsoidal asteroid. Two of the semiaxes are equal to $A$, and the third is equal to $B \gg A$. The asteroid rotates about one of the short axes. Two extreme cases may occur: (1) the asteroid is viewed pole-on and the light fluctuations are minimum and (2) the rotation axis of the asteroid is perpendicular to the line of sight and the light variations are maximum. Also, suppose that a spherical planet of the same material and surface macrostructure has a phase coefficient $\beta_{\text {sphere }}$.

In case (2), at maximum light, the situation is identical to case (1) and

$$
\beta_{\max }=\beta_{(1)}<\beta_{\text {sphere }}
$$

The inequality follows from the fact that on the ellipsoid, at maximum light, the average $i$ and $\epsilon$ are effectively smaller than on the sphere, and the effects of shadowing are therefore less important. However, at minimum light, the average $i$ and $\epsilon$ are effectively larger than in the case of a sphere and therefore shadowing is more important. Hence

$$
\beta_{\min }>\beta_{\text {sphere }}>\beta_{(1)}
$$

Usually, in case (2), $\beta$ would be determined by using the mean magnitude of the lightcurve, so that

$$
\beta_{(2)} \equiv \frac{\beta_{\max }+\beta_{\min }}{2}>\beta_{(1)}
$$

Therefore, it is possible to predict that for an irregular asteroid whose aspect changes with time and whose surface is macroscopically rough:

(1) The apparent $\beta$ is largest when the amplitude of the lightcurve is maximum.

(2) If the aspect of an asteroid stays approximately constant during an opposition, then the phase coefficient determined from the minima of the lightcurve should be larger than that determined from the maxima; that is

$$
\beta_{\min }>\beta_{\max }
$$


Thus, to even meaningfully define a phase coefficient for a very irregular asteroid whose aspect changes significantly with time may require a long series of accurate observations.

\section{CONCLUSIONS}

In summary, the situation appears bleak. One cannot expect to derive the geometric albedos of asteroids from their phase coefficients. The contrary claim by Widorn (1964) and others is largely based on a fortuitous empirical relationship obtained by plotting $\beta$ against $p$ for the Moon and some of the large planets. Jupiter and Venus are intrinsically bright (large $p$ ) and have cloud decks in which multiple scattering is important (low $\beta$ ). Mercury and the Moon are intrinsically dark (low $p$ ) and have rough dark surfaces (high $\beta$ ). Thus one can arrive at the unfounded conclusion that $\beta$ must always be inversely correlated with $p$, which in the case of dark surfaces certainly need not be true.

Because the degree of surface roughness ( $Q$ in the above model) of any particular asteroid is not known, one cannot convert an observed phase coefficient $\beta$ in its laboratory counterpart $\beta_{\text {lab }}$. Furthermore, even if this were possible, little diagnostic information could be obtained from $\beta_{\text {lab }}$. (See the previous discussion of $\beta_{\text {lab }}$.)

In addition, for very irregular asteroids with rough surfaces it may be difficult to even define a meaningful phase coefficient (as discussed in the preceding section). Fortunately, there are some asteroids, Ceres and Flora, for example, that are almost spherical, so that at least this complication does not arise. Flora has a phase coefficient similar to that of the Moon: $0.028 \mathrm{mag} / \mathrm{deg}$ in $V$ (Veverka, 1971). If it is composed of photometrically similar material, its surface roughness must also be similar. If it is rougher than the Moon, its surface material must be less backscattering, and vice versa. The phase coefficient of Ceres, $0.050 \mathrm{mag} / \mathrm{deg}$ in $V$ (Ahmad, 1954), is considerably larger than that of the Moon. This is probably not entirely due to surface roughness because, as figure $4(b)$ shows, for lunarlike materials it is difficult to increase $\beta$ beyond $0.05 \mathrm{mag} / \mathrm{deg}$ by increasing surface roughness. This suggests that the surface material of Ceres is intrinsically more backscattering than that of the Moon.

According to the above model the color dependence of asteroid phase coefficients should be small. This does seem to be the case. For Vesta, for example, $\beta_{V}=0.0253 \mathrm{mag} / \mathrm{deg}, \beta_{B}=0.0264 \mathrm{mag} / \mathrm{deg}$, and $\beta_{U}=0.0291$ $\mathrm{mag} / \mathrm{deg}$ (Gehrels, 1967). Because the reflectivity of Vesta increases with increasing wavelength in the $U B V$ region of the spectrum, this slight decrease in $\beta$ with increasing wavelength may perhaps be attributed to the increased importance of the small, multiply scattered component at long wavelengths, as suggested above. If this is true, then the wavelength dependence of asteroid phase coefficients mostly contains information about the wavelength dependence of the surface reflectivity, information that can be obtained more easily from a single spectral reflectivity measurement. 
Finally, I would like to stress again that typical asteroid phase coefficients $(0.025$ to $0.035 \mathrm{mag} / \mathrm{deg})$ cannot be interpreted unambiguously. This is because the observed phase coefficient may depend as much on the photometric properties of an individual surface element $f(\alpha, D)$ as on the degree of large-scale roughness $\Sigma(\alpha, Q)$. (See preceding discussions of these functions.) If only disk integrated measurements of the scattered light are available, these two effects cannot be separated. In spite of this, there does seem to be some point in looking for objects with unusual phase coefficients, such as Ceres.

In some cases, it should be possible to estimate the relative surface roughness of two quasi-spherical asteroids by combining photometric and polarimetric observations. For example, if the two asteroids have almost identical polarization curves, but quite different phase coefficients, it is likely that the asteroid with the larger phase coefficient has a macroscopically rougher surface.

\section{ACKNOWLEDGMENTS}

I wish to thank C. Sagan and F. L. Whipple for their advice and generous assistance. The numerical calculations described in this paper were carried out at the Smithsonian Astrophysical Observatory, and I am grateful to the Smithsonian Foundation for support during this phase of the project. This work was also supported in part by NASA NGR 33-010-082.

\section{REFERENCES}

Ahmad, I. I. 1954, Photometric Studies of Asteroids. IV. The Light Curves of Ceres, Hebe, Flora, Kalliope. Astrophys. J. 120, 551.

Beckmann, P. 1965, Shadowing of Random Rough Surfaces. IEEE Trans. Antennas Propagat. Ap-13, 384.

Bell, L. 1917, The Physical Interpretation of Albedo. Astrophys. J. 45, 1.

Beresford, R. H. 1969, Statistical Geometry of Random Heaps of Equal Hard Spheres. Nature 224, 550.

Gehrels, T. 1956, Photometric Studies of Asteroids. V. The Light Curve and Phase Function of 20 Massalia. Astrophys. J. 123, 331.

Gehrels, T. 1967, Minor Planets. I. The Rotation of Vesta. Astron. J. 72, 929.

Gehrels, T., Roemer, E., Taylor, R. C., and Zellner, B. H. 1970, Minor Planets and Related Objects. IV. Asteroid (1566) Icarus. Astron. J. 75, 186.

Halajian, J. D. 1965, Photometric Measurements of Simulated Lunar Surfaces. Grumman Res. Dept. Rept. RE-219.

Halajian, J. D., and Spagnolo, F. J. 1966, Photometric Measurements of Simulated Lunar Surfaces. Grumman Res. Dept. Rept. RE-245.

Hämeen-Anttila, K. A., Laakso, P., and Lumme, K. 1965, The Shadow Effect in the Phase Curves of Lunar Type Surfaces. Ann. Acad. Sci. Fenn. Ser. A. 172.

Hapke, B. W. 1963, A Theoretical Photometric Function for the Lunar Surface. J. Geophys. Res. 68, 4571.

Henyey, L. G., and Greenstein, J. L. 1941, Diffuse Reflection in the Galaxy. Astrophys. J. 93, 70.

Horak, H. G. 1950, Diffuse Reflection by Planetary Atmospheres. Astrophys. J. 112, 445. 
Irvine, W. M. 1966, The Shadow Effect in Diffuse Reflection. J. Geophys. Res. 71, 2931. Saunders, P. M. 1967, Shadowing on the Ocean and the Existence of the Horizon. J. Geophys. Res. 72, 4643.

Stumpff, K. 1948, Concerning the Albedos of Planets and the Photometric Determination of Diameters of Asteroids. Astron. Nachr. 276, 118.

Veverka, J. 1971, Photopolarimetric Observations of the Minor Planet Flora. Icarus 15, 11.

Widorn, T: 1964, Phase Coefficients and Phase Integrals. Mitt. Wien Sternw. 12, 17.

\section{DISCUSSION}

HAPKE: It is very likely true, as Veverka has stated, that one could not make a phase function as steep as Ceres' with lunar soil roughened in some fashion. However, the following conceptual model shows that it is possible to construct a surface with a phase function as steep as one wishes. Imagine a body with a relatively smooth surface; such a body would have a phase function something like that corresponding to Lambert's law. Now replace the smooth surface by large dark particles. Since the phase function of the body is given by the product of the phase function of the particles and their shadowing function, the body phase function will be steepened. Next, replace the particles by clumps of smaller particles. Now the phase function of the body involves a product: the phase function of the smaller particles times that of the clumps times the shadowing function. The body phase function is even steeper. This procedure can be followed until the diffraction limit for casting sharp shadows is reached. Such a hierarchy of clumps of material similar to lunar soil may not be a completely unreasonable model in view of the very low surface gravity on asteroids. 